\title{
Changes in Berry Firmness of Various Grape Cultivars on Vines Measured by Nondestructive Method before and after Veraison
}

\author{
Masahiro Takahashi $^{1 *}$, Mitsuru Taniwaki ${ }^{2}$, Naoki Sakurai ${ }^{1}$, Toshihito Ueno ${ }^{3 * *}$ \\ and Hiroshi Yakushiji ${ }^{3}$ \\ ${ }^{1}$ Graduate School of Biosphere Science, Hiroshima University, Higashi-Hiroshima 739-8521, Japan \\ ${ }^{2}$ Collaborative Research Center, Hiroshima University, Higashi-Hiroshima 739-8527, Japan \\ ${ }^{3}$ Persimmon and Grape Research Center, National Institute of Fruit Tree Science, Higashi-Hiroshima 739-2494, Japan
}

Time-course changes in the elasticity index (EI) of 8 cultivars and 1 selection of grape berries on vines were investigated with a nondestructive acoustic method. A portable device was constructed for the measurement of grape firmness. The $\mathrm{EI}$ was determined by the formula $\mathrm{EI}=f_{2}{ }^{2} \cdot d^{2}$, where $f_{2}$ and $d$ were the second resonant frequency and the diameter of the sample respectively. In all cultivars, the date of veraison was judged by touch and the EI largely decreased around veraison. These changes were classified into two types; one decreased before veraison and the other after veraison. The EI was compared with the firmness measured by two penetration methods. Relationships between the EI and firmness measured by the penetration methods were classified into three types of regression curves; a linear relation which detected similar berry firmness with both the nondestructive method and the penetration methods; logarithmic, in which the nondestructive method was more sensitive when berries were hard; and exponential, in which the nondestructive method waas more sensitive when berries were soft. We concluded that these differences were caused by differences in the spatial softening process.

Key Words: firmness, grape berry, nondestrucitve measurement, ripening, veraison.

\section{Introduction}

Firmness is related to the degree of maturation of fruits of many kinds (Taniwaki and Sakurai, 2010). Grape berries show characteristic changes in firmness through ripening, and veraison is the period when berry firmness falls markedly. It is generally recognized that firmness changes of fruit or plant tissue are attributed to changes in the mechanical properties of the cell walls; therefore, pre- and post-veraison, changes in cell wall components of berries have been reported. For example, Nunan et al. (1998) revealed the alteration of specific polysaccharide components and protein composition in the cell walls of ripening grape berries. Subsequently, Yakushiji et al. (2001) reported depolymerization of pectin and xyloglucan, and a decreased amount of cellulose related to softening of the grape berry. Deytieux-Belleau et al. (2008) suggested that pectin methylesterase and

Received; October 16, 2009. Accepted; May 11, 2010.

* Corresponding author (E-mail: masahirot@hiroshima-u.ac.jp).

** Present address: Yamanashi Fruit Tree Experimental Station, Yamanashi 405-0043, Japan polygalacturonase contribute to the softening of berry skin. Thomas et al. (2006) measured turgor of berries and revealed that turgor decreased along the ripening stages of grapes. These biochemical and physiological changes in the berry are supposed to be related to firmness changes through grape maturation.

Little attention, however, has been paid to examining the firmness of berries on vine. Sato et al. $(1997,2004)$ developed a method for measuring the texture of harvested berries using a penetration plunger; however, firmness measured by penetration methods is destructive and reflects local features depending on the penetration point or features of the plunger. If whole berry firmness can be nondestructively measured on the vine, changes in individual berry firmness will afford more precise information to understand the mechanism of berry fruit firmness and the index of its maturity.

Nondestructive acoustic vibration techniques have been widely adopted to measure the firmness of many kinds of fruits, since Cooke (1972) reported that an index determined by the second lowest resonant frequency and the mass of a test object can be used as an elasticity index of a spherical object. For instance, these techniques 
have precisely revealed the ripening process of kiwifruits (Muramatsu et al., 1997a; Terasaki et al., 2001), 'La France' pears (Terasaki et al., 2006), and melons (Taniwaki et al., 2009). Terasaki et al. (2006) measured the changes in elasticity index of 'La France' pears under cold storage condition by a nondestructive method using a laser Dopplar vibrometer and identified biphasic decay in the elasticity index. Kuroki et al. (2006) developed a portable device with piezoelectric sensors for the measurement of second resonant frequency and monitored the firmness changes of melons growing in a greenhouse. These methods could provide continual measurement of fruits firmness.

The aims of this study were to develop a new portable vibration detector to measure the firmness of smaller fruits, grape berries, to identify precise changes in berry firmness before and after veraison, and to compare nondestructive measurements with conventional destructive measurements. The firmness changes of 8 cultivars and 1 selection of grape on the vine were used to analyze different types of firmness changes among varieties. The results were compared with measurements using two conventional penetration methods.

\section{Materials and Methods}

\section{Samples}

Eight cultivars and one selection of grape berries (Table 1) grown in an orchard (Persimmon \& Grape Experimental Station, Akitsu, Hiroshima, Japan) in 2007 were used as experimental materials. For seedless cultivation, two gibberellin $\left(\mathrm{GA}_{3}\right)$ treatments are carried out at the full bloom stage and 10-15 days after full bloom. The date of veraison for each cultivar was judged using a thumb and forefinger.

For nondestructive acoustic measurement of berry firmness, five bunches of grapes were selected for each cultivar. One upper and one lower berry of each bunch were used for the firmness measurement. Since no significant difference between the firmness of upper and bottom berries was found, the mean value of the two was used as the representative firmness value for each bunch of grapes. In this measurement, identical berries were measured consistently. Five to ten berries were randomly selected for the two types of penetration tests.

\section{Nondestructive acoustic measurement}

A portable device for resonance measurement was developed for nondestructive evaluation of the firmness of grape berries (Fig. 1). A piezoelectric vibration generator was attached to the thumb and a piezoelectric vibration detector to the forefinger. A grape berry was sandwiched between them.

The sample was mechanically excited by the vibration generator driven by swept sine wave signals $(0-5 \mathrm{kHz}$ for $10 \mathrm{~s}$ ) generated by a PC. The vibrations of the samples were detected by the vibration detector and the

Table 1. Veraison of grape cultivars and selection.

\begin{tabular}{lccc}
\hline \hline Cultivar & Seed & Date of veraison & Date of harvest \\
\hline Kyoho & seedless & Jul. 17, 2007 & Sep. 1, 2007 \\
Shine Muscat & seedless & Jul. 20, 2007 & Sep. 1, 2007 \\
Oriental Star & seedless & Jul. 26, 2007 & Sep. 12, 2007 \\
Akitsu 25 & seedless & Jul. 26, 2007 & Sep. 3, 2007 \\
Honey Venus & - & Jul. 17, 2007 & Sep. 3, 2007 \\
North Red & - & Jul. 17, 2007 & Sep. 3, 2007 \\
Campbell Early & - & Jul. 20, 2007 & Aug. 23, 2007 \\
Delaware & - & Jul. 26, 2007 & Sep. 12, 2007 \\
Kyoho & - & Jul. 26, 2007 & Sep. 8, 2007 \\
Sekirei & - & Aug. 1, 2007 & Sep. 12, 2007 \\
\hline
\end{tabular}

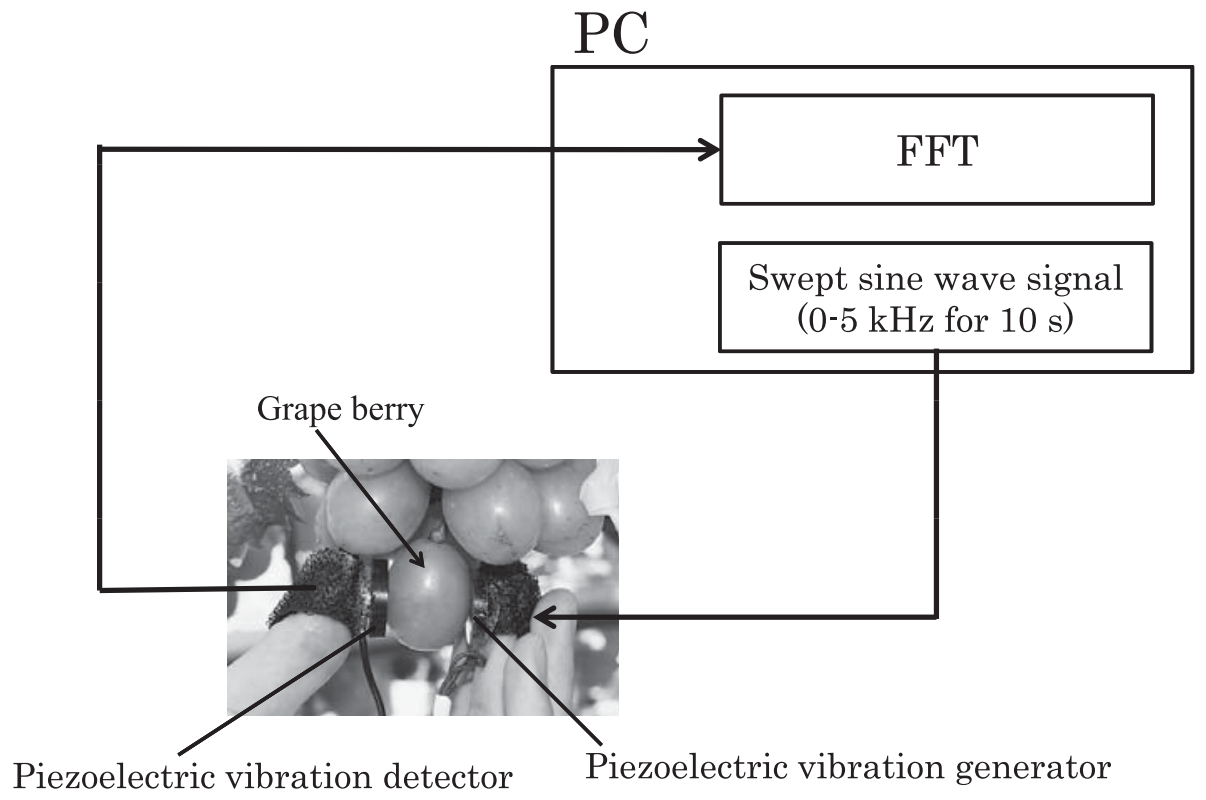

Fig. 1. Diagram of setup for the non-destructive measurement of grape berry firmness and field measurement. FFT, Fast Fourier Transformation. 
corresponding voltage signals were transferred to the PC. The resonant frequencies of the samples were determined by fast Fourier transformation (FFT) of the acquired signals.

The EI was originally used as the firmness index, according to the following formula (Cooke, 1972; Terasaki et al., 2001):

$$
\mathrm{EI}=f_{2}^{2} \cdot m^{2 / 3}
$$

where $f_{2}(\mathrm{~Hz})$ is the second lowest resonant frequency and $m(\mathrm{~kg})$ is the mass of a sample. Since the mass was not easily measured in the case of grape berries on the vine, the diameter of the sample measured by a slide caliper was used as a substitute for the mass, i.e., EI was estimated according to the following formula in this measurement:

$$
\mathrm{EI}=f_{2}^{2} \cdot d^{2}
$$

where $d(\mathrm{~m})$ is the diameter of the samples.

\section{Penetration test}

Two penetration tests were used to evaluate the firmness of each grape cultivar and the results were compared with the EI.

\section{(i) Universal hardness meter}

A universal hardness meter (KM-1; Fujiwara Scientific Company, Ltd., Tokyo, Japan) was used for the penetration test. It has a $5 \mathrm{~mm}$ diameter plunger and determines the stiffness of samples by the maximum penetration force. Five to ten berries were sampled from each cultivar and cut in half at the equatorial plane. The plunger was inserted into the mesocarp by hand and the maximum force was recorded.

\section{(ii) Texture analyzer}

In addition to the universal hardness meter, a texture analyzer (TPU-2S; Yamaden Co. Ltd., Tokyo, Japan) was used for evaluating grape berry firmness. Five to ten berries were sampled from each cultivar and sliced into $8 \mathrm{~mm}$ sections around the equatorial plane. The plunger $(\phi 2 \mathrm{~mm})$ was inserted into the mesocarp to a depth of $6 \mathrm{~mm}$ at the speed of $10 \mathrm{~mm} \cdot \mathrm{s}^{-1}$. The maximum penetration force was used as the firmness of the samples.

\section{Results}

\section{Reliability test of the portable device}

The reliability of the portable device was verified by comparing the resonant frequencies of two rubber balls (A, $47.5 \mathrm{~mm} ; \mathrm{B}, 36.3 \mathrm{~mm}$ in diameter) measured by this device and a laser Doppler vibrometer (LDV) method. The LDV is a widely used definitive device for measuring the frequency response of vibrating materials (Muramatsu et al., 1997b). The second lowest resonant frequencies of the rubber balls were measured five times by each method. The second resonant frequency of ball A was $559.0 \pm 0.0 \mathrm{~Hz}(\mathrm{CV}=\mathrm{SD} / \mathrm{Mean}=0.00 \%)$ for the LDV method, and $575.4 \pm 1.5 \mathrm{~Hz}(\mathrm{CV}=0.26 \%)$ for the portable device. The second resonant frequency of ball
B was $758.0 \pm 1.2 \mathrm{~Hz}$ for the LDV method (CV $=0.16 \%)$, and $774.6 \pm 3.8 \mathrm{~Hz}(\mathrm{CV}=0.49 \%)$ for the portable device. Measurement with the portable device gave $16.4 \mathrm{~Hz}$ $(2.9 \%)$ for ball $\mathrm{A}$, and $16.6 \mathrm{~Hz}(2.2 \%)$ for ball $\mathrm{B}$, higher than that with the LDV method.

Moreover, the reliability of the portable device was tested by comparing the second lowest frequencies of three cherry tomatoes (A, $28.9 \mathrm{~mm}$; B, $26.2 \mathrm{~mm}$; C, $29.7 \mathrm{~mm}$ in diameter) using the same methods. The second resonant frequencies by LDV were $1200.8 \pm$ $0.9 \mathrm{~Hz}(\mathrm{CV}=0.16 \%)$ for $\mathrm{A}, 1052.8 \pm 1.6 \mathrm{~Hz}(\mathrm{CV}=0.34 \%)$ for $\mathrm{B}$, and $1163.0 \pm 2.1 \mathrm{~Hz}(\mathrm{CV}=0.39 \%)$ for $\mathrm{C}$. The second resonant frequencies by the portable device were $1231.0 \pm 5.0 \mathrm{~Hz}(1.21 \%)$ for $\mathrm{A}, 1062.80 \pm 5.0 \mathrm{~Hz}(\mathrm{CV}=$ $1.06 \%)$ for $\mathrm{B}$, and $1185.2 \pm 6.3 \mathrm{~Hz}(\mathrm{CV}=1.20 \%)$ for $\mathrm{C}$. Measurement with the portable device was $20.8 \pm 5.9 \mathrm{~Hz}$ higher than with the LDV method.

\section{Changes in the elastic index of grapes on the vine}

Figure 2 shows the time-course changes in the EI of eight grape cultivars and one selection. Arrows in the figure represent the onset of veraison determined by touching the grapes with a thumb and forefinger. As time elapsed, the EI largely decreased in all cultivars. The EIs of 'North Red' and 'Campbell Early' at the beginning of the measurement (July 11) were significantly higher than those of other varieties and higher than those measured on July 17 around veraison. This was also the case for 'Kyoho' and 'Akitsu 25'. A continual decrease in the EI from July 11 to the end of the experiment on August 8 was found for 'Honey Venus'; however, the EI of some cultivars increased or remained high before veraison. In the case of 'Sekirei' and 'Oriental Star', the EIs measured on July 11 were lower than those measured on July 17. For 'Akitsu 25', 'Kyoho', 'Delaware', and 'Shine Muscat', the EIs on July 11 remained at a similar level on July 17 . The varieties with increased or maintained high EIs showed relatively late veraison after July 25 , as compared with 'Kyoho (seedless)' and 'North Red' for which the date of veraison was July 17. 'Kyoho (seedless)' and 'North Red' might have exhibited higher EI before the start of this experiment.

To clarify such characteristic changes in the EI before and after veraison, the data were normalized in terms of the date of veraison (Fig. 3). This normalization revealed that the grape varieties could be classified into two types in terms of the EI changes: one decreasing EI before veraison (type $A$ ) and the other after veraison (type B). The EI of type A-1, -2, and -3 in Figure 3 decreased significantly before veraison. Type A included 'Akitsu25', 'Sekirei', 'Kyoho', 'Kyoho (seedless)', 'North Red', and 'Campbell Early'. The EI of 'Campbell Early' was not measured near veraison; however, it was classified as type A, because it exhibited EI changes quite similar to those of 'North Red'. On the other hand, 'Oriental Star', 'Delaware', 'Shine Muscat', and 'Honey 


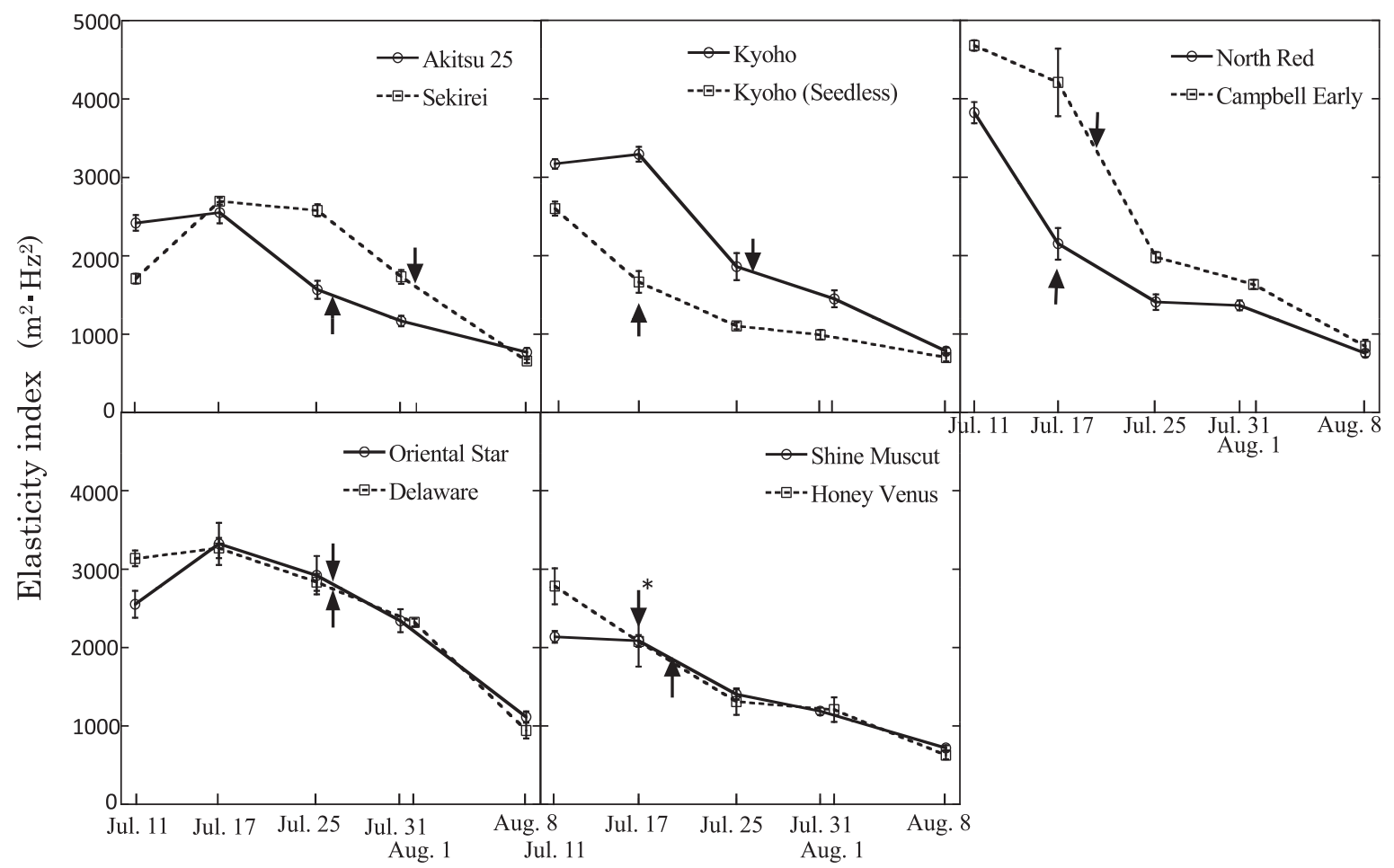

Date (2007)

Fig. 2. Time-course changes in the elasticity index (EI) measured by the nondestructive acoustic method. Arrows represent the date of veraison for each cultivar judged by touch. Vertical bars indicate SE $(n=10)$.

* Veraison of Honey Venus.

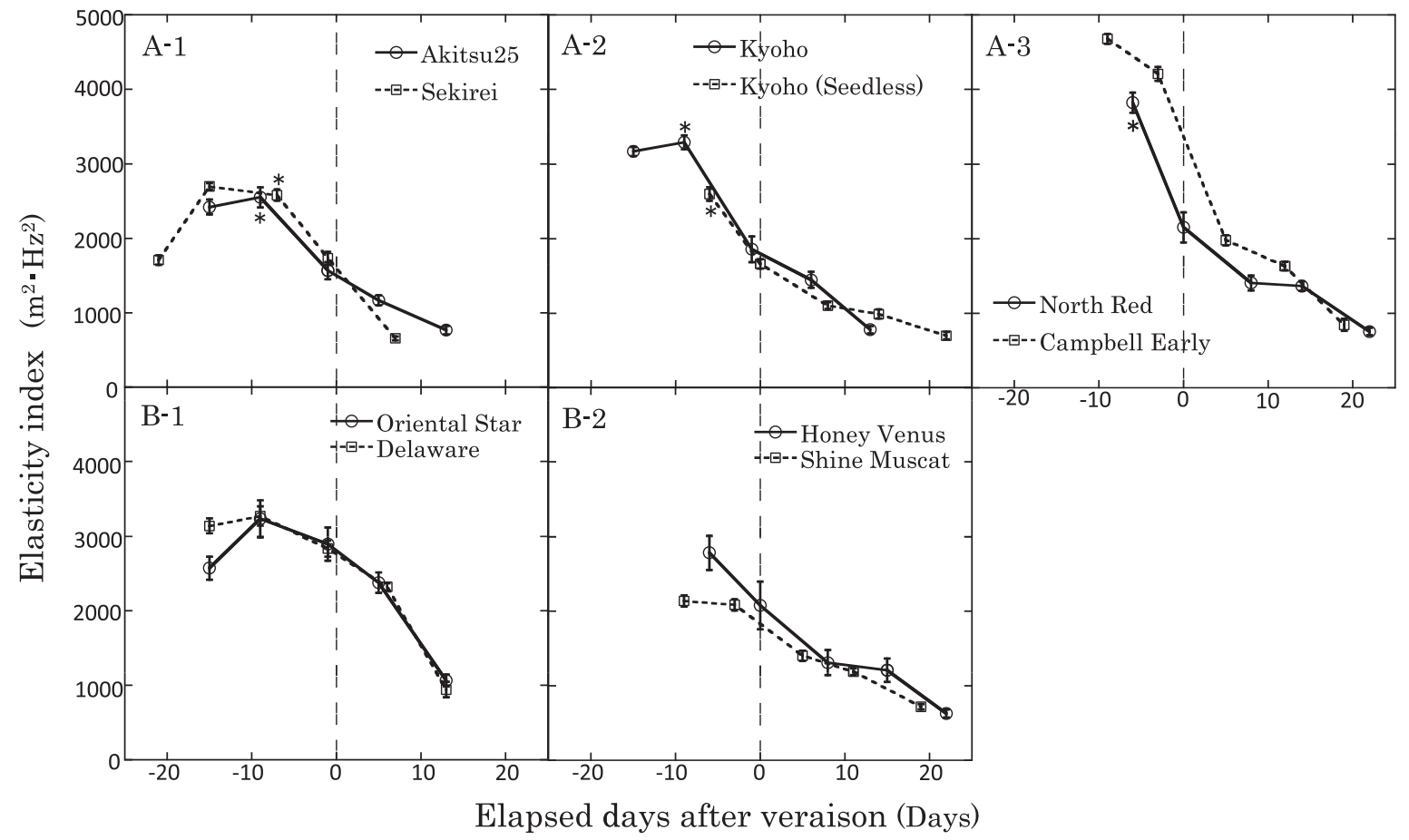

Fig. 3. Time-course changes in the elasticity index (EI) normalized in terms of the date of veraison. Vertical broken lines represent the date of veraison.

* Significant difference before veraison at $1 \%$ level by $t$-test. 
Venus' belong to type B, since more remarkable decrease in EI was observed after veraison.
Comparison between the nondestructive and two destructive methods

Figures 4 and 5 show the relationship between the EI determined by the nondestructive acoustic method and

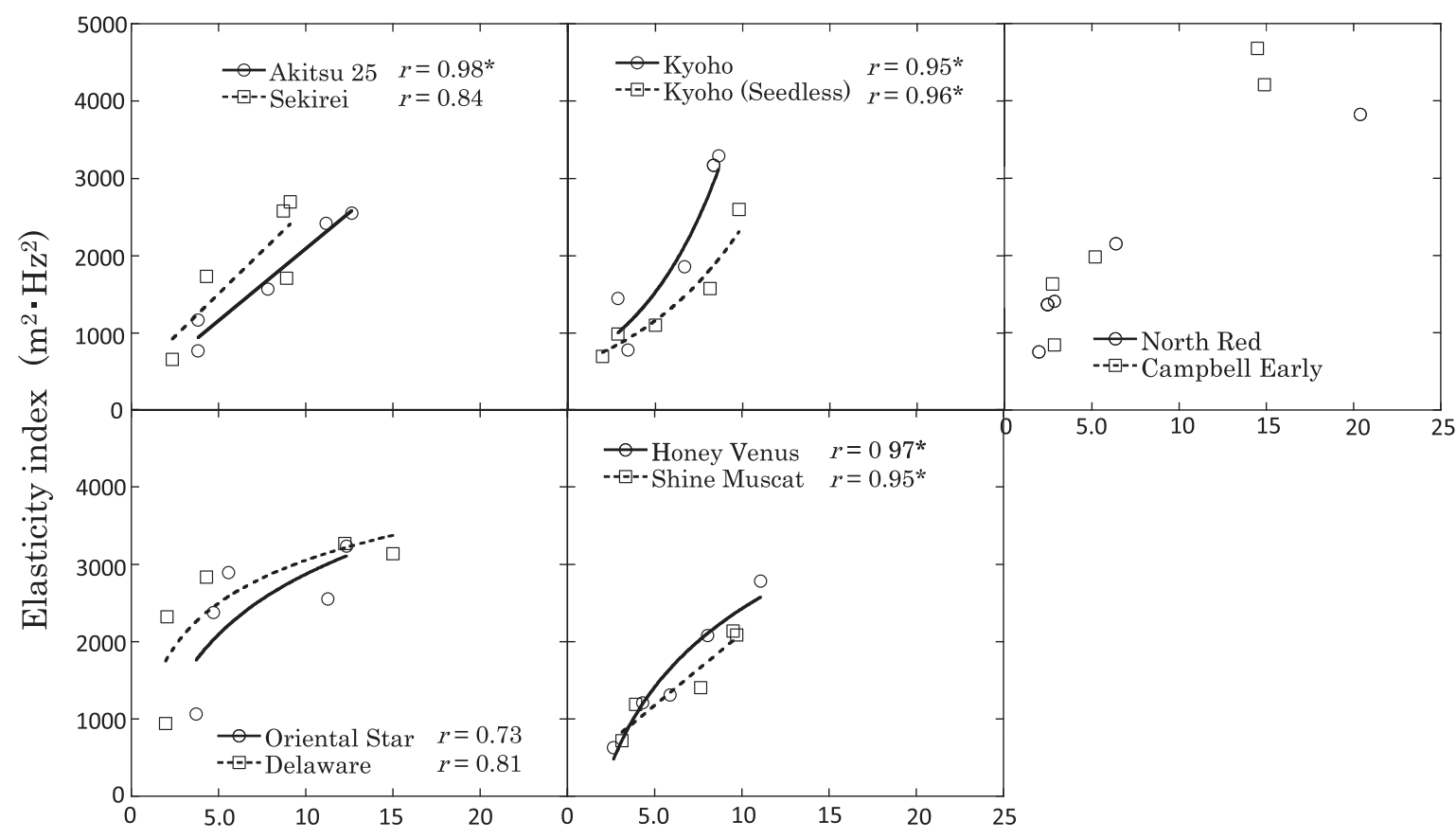

Maximum penetration force by a universal hardness meter $\left(\mathrm{N} \cdot \mathrm{m}^{-2}\right)$

Fig. 4. Relationship between the elasticity index (EI) measured by the nondestructive acoustic method and the maximum penetration force measured by the universal hardness meter.

* Significance at the $5 \%$ level by $t$-test.

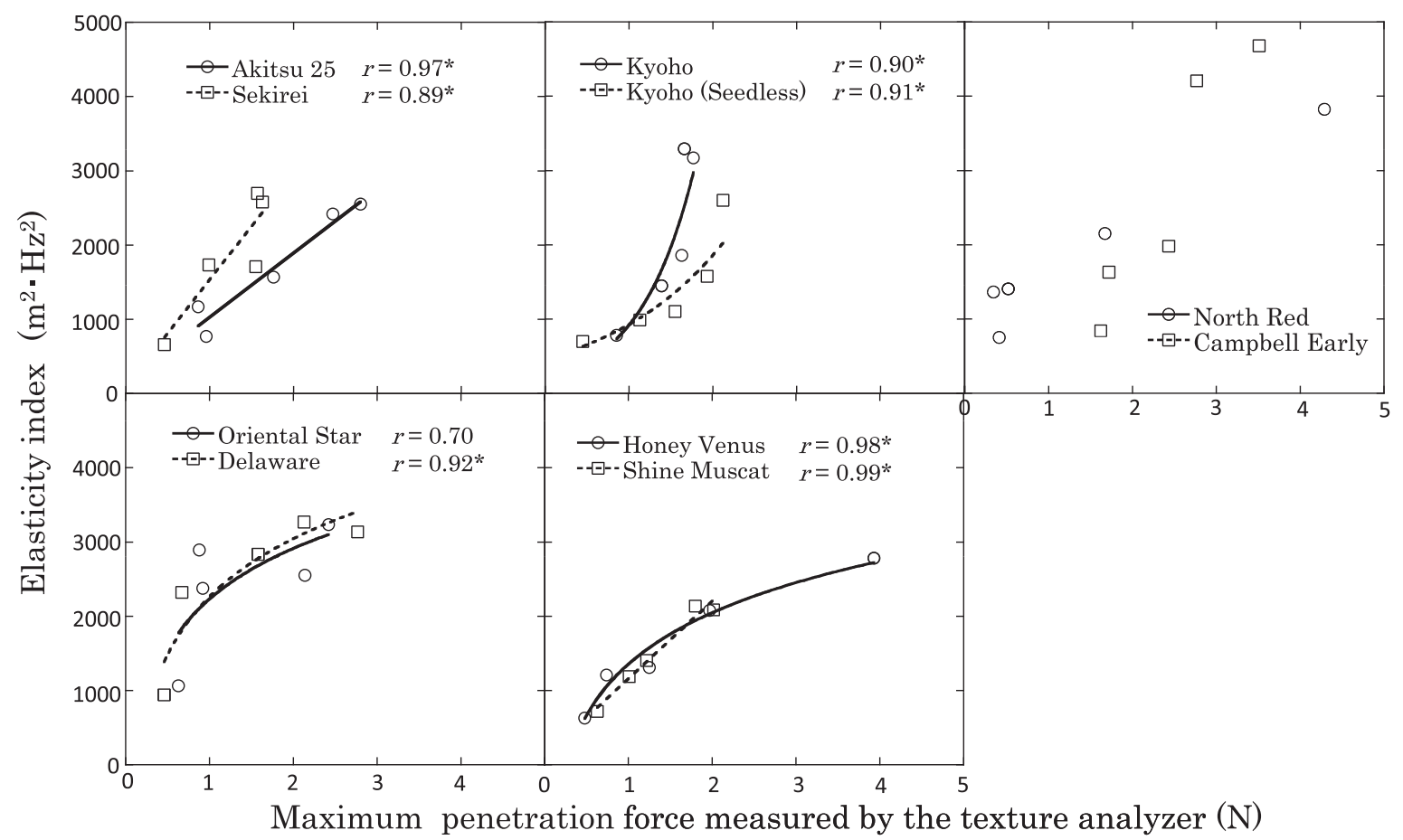

Fig. 5. Relationship between the elasticity index (EI) measured by the nondestructive acoustic method and the maximum penetration force measured by the texture analyzer.

* Significance at the $5 \%$ level by $t$-test. 
the maximum penetration force measured by two destructive methods. These destructive tests were performed on the same day as the nondestructive measurement or the next morning. The maximum penetration force did not always linearly correlate with those measured by the nondestructive method. The relationships were classified into three types in terms of the relation pattern: linear ('Sekirei', 'Akitsu25', 'North Red', 'Campbell Early', and 'Shine Muscat'), logarithmic ('Oriental Star', 'Delaware', and 'Honey Venus') and exponential ('Kyoho' and 'Kyoho (Seedless)'). In North Red and Campbell Early, the limited number of data may not show a clear relationship. The logarithmic correlation suggests that elasticity changes in the late stage of grape development that were not detected by destructive methods were able to be detected by the nondestructive acoustic method. The exponential correlation suggests that firmness changes in the late stages that were difficult to detect by the nondestructive acoustic method were able to be evaluated by destructive methods.

\section{Discussion}

\section{Firmness measurement of growing grape berries}

Continual and nondestructive measurements of timecourse changes in the firmness of growing grape berries on the vine before and after veraison were first conducted by a new portable acoustic device. The firmness of grape berries was evaluated by the EI using our portable device for acoustic vibration measurement. Generally, EI of harvested fruits is calculated by $f_{2} \cdot \mathrm{m}^{2 / 3}$, where $m$ is the mass of fruits and $f_{2}$ the second resonant frequency. In this study, the EI was determined by the second resonant frequency and the diameter of the grape berries because it was impractical to measure the mass of each berry on the vine. Two-third power of mass is equivalent to the square of the diameter in relative scale.

\section{Reliability of the portable device}

The reliability of the new portable device was examined by measuring the resonant frequency of two rubber balls and three cherry tomatoes. The results showed that the device was precise enough for practical purposes because the $\mathrm{CV}$ (SD/Mean) was less than $0.49 \%$ for rubber balls and $1.21 \%$ for tomatoes, although the CV for the method using an LDV was even smaller. The reason why the second resonant frequencies measured with the portable device were higher than those by the LDV remained unknown, but the force required to sandwich the ball possibly led to an increase in the elasticity of the balls and tomatoes, resulting in a higher resonant frequency. This is similar to an effect reported by Muramatsu et al. (1997b): the slight difference in resonant frequencies between measurements with an accelerometer and an LDV was due to the small weight (more than $5 \mathrm{~g}$ ) of the accelerometer placed on the object.
Comparison between the EI and the date of veraison

Veraison is a characteristic stage of grape berry growth. Notable features of veraison are color development and a marked decrease in firmness. In this measurement, the date of veraison for each cultivar was determined by touch; however, we noticed a decrease in firmness of some cultivars before veraison. Moreover, the results showed that the EI of some examined grape cultivars increased before veraison. In four cultivars ('Akitsu 25', 'Kyoho', 'Oriental Star', and 'Delaware'), the EI increased until about ten days before veraison, i.e. the EI peaked about ten days before veraison. It can be assumed that the other six cultivars might also have exhibited increases in the EI before veraison if they had been measured before July 11 .

This time difference is probably due to the fact that judging by the thumb and forefinger gives the surface firmness of a fruit, while the acoustic vibration technique gives the average firmness of the whole fruit. These results suggest that acoustic measurement determines the precise date of veraison.

Figure 3 also shows that both Kyoho normal and seedless cultivations changed their EI very similarly. Sato et al. (1997) reported no significant effect of gibberelic acid on the firmness of growing grape berries. Our results support their data and suggest that the formation of seeds does not affect whole berry firmness.

Comparison between the EI and the firmness measured using conventional destructive methods

Figures 4 and 5 revealed that the relation between the nondestructively determined EI and the result of two destructive tests could be classified into three types in terms of the pattern of regression curves: linear, logarithmical, and exponential. The linear relation suggests that both nondestructive and destructive methods detected similar berry firmness. In 'North Red' and 'Campbell Early', more samples are necessary to show their linear relation. The logarithmic relation means that destructive methods are more sensitive when the berries are hard, while the non-destructive method is more sensitive when the berries are soft. In contrast, the exponential type implies that the nondestructive method is more sensitive when berries are hard, while destructive methods are more sensitive than the nondestructive method when berries are soft. Such characteristics are probably attributed to the difference in sensitivity for the detection of firmness. Destructive methods measure the outer mesocarp, while the nondestructive acoustic method probably detects the average firmness of the berries. From this point of view, the firmness of the cultivars showing an exponential relation seemed to begin to decrease in the central part of a berry, and the firmness of those showing logarithmic relations began to decrease near the exterior. The second resonant frequency is supposed to reflect physical properties near the center of an object. On the other hand, the probes 
used for the destructive methods penetrated the outer mesocarp of the berries; therefore, such a difference may have caused a different softening process in cultivars. Further detailed study of the particular softening process of the grape berry mesocarp is necessary.

\section{Literature Cited}

Cooke, J. R. 1972. An interpretation of the resonant behavior of intact fruits and vegetables. Trans. ASAE. 15: 1075-1080.

Deytieux-Belleau, C., A. Vallet, B. Doneche and L. Geny. 2008. Pectin methylesterase and polygalacturonase in the developing grape skin. Plant Physiol. Biochem. 46: 638-646.

Kuroki, S., M. Tohro and N. Sakurai. 2006. Monitoring of the elasticity of melon fruit in a green house. J. Japan. Soc. Hort. Sci. 75: 415-420.

Muramatsu, N., N. Sakurai, N. Wada, R. Yamamoto, T. Takahara, T. Ogata, K. Tanaka., T. Asakura, Y. Ishikawa-Takano and D. J. Nevins. 1997b. Critical comparison of an accelerometer and a laser Doppler detection. HortTechnol. 7: 434-438.

Muramatsu, N., N. Sakurai, R. Yamamoto, D. J. Nevins, T. Takahara and T. Ogata. 1997a. Comparison of a nondestructive acoustic method with an intrusive method for firmness measurement of kiwifruit. Postharvest Biol. Technol. 12: $221-228$.

Nunan, J. K., I. M. Sims, A. Bacic, S. P. Robinson and G. B. Fincher. 1998. Changes in cell wall composition during ripening of grape berries. Plant Physiol. 118: 783-792.

Sato, A., M. Yamada, H. Iwanami and N. Mitani. 2004.
Quantitative measurements of grape fresh texture as affected by gibberellic acid application. J. Japan. Soc. Hort. Sci. 73: $7-11$.

Sato, A., H. Yamane, N. Hirakawa, K. Otobe and M. Yamada. 1997. Varietal differences in the texture of grape berries measured by penetration tests. Vitis $36: 7-10$.

Taniwaki, M. and N. Sakurai 2010. Evaluation of the internal quality of agricultural products using acoustic vibration technique. J. Japan. Soc. Hort. Sci. 79: 113-128.

Taniwaki, M., M. Takahashi and N. Sakurai. 2009. Determination of optimum ripeness for edibility of postharvest melons using nondestructive vibration. Food Res. Int. 42: 137-141.

Terasaki, S., N. Sakurai, J. Zebrowski, H. Murayama, R. Yamamoto and D. J. Nevins. 2006. Laser Doppler vibrometer analysis of changes in elastic properties of ripening 'La France' pears after postharvest storage. Postharvest Biol. Technol. 42: 198207.

Terasaki, S., N. Wada, N. Sakurai, N. Muramatsu, R. Yamamoto and D. J. Nevins. 2001. Nondestructive measurement of kiwifruit ripeness using a laser Doppler vibrometer. Trans. ASAE. 44: 81-87.

Thomas, R. T., M. A. Matthews and K. A. Shackel. 2006. Direct in situ measurement of cell turgor in grape (Vitis vinifera L.) berries during development and in response to plant water deficits. Plant Cell Environ. 29: 993-1001.

Yakushiji, H., N. Sakurai and K. Morinaga. 2001. Changes in cellwall polysaccharides from the mesocarp of grape berries during veraison. Physiol. Plant. 111: 188-195. 